\title{
Direct Entry of Gadolinium into the Vestibule Following Intratympanic Applications in Guinea Pigs and the Influence of Cochlear Implantation
}

\author{
E. B. King ${ }^{1}$, A. N. SAlt $^{2}$, H. T. Eastwood ${ }^{1}$, and S. J. O'Leary ${ }^{1}$ \\ ${ }^{1}$ Department of Otolaryngology, University of Melbourne, 32 Gisborne Street, East Melbourne, VIC 3002, Australia \\ ${ }^{2}$ Washington University School of Medicine, St. Louis, MO, USA
}

Received: 14 February 2011; Accepted: 16 June 2011; Online publication: 19 July 2011

\begin{abstract}
Although intratympanic (IT) administration of drugs has gained wide clinical acceptance, the distribution of drugs in the inner ear following IT administration is not well established. Gadolinium (Gd) has been previously used as a marker in conjunction with magnetic resonance imaging (MRI) to visualize distribution in inner ear fluids in a qualitative manner. In the present study, we applied gadolinium chelated with diethylenetriamine penta-acetic acid (Gd-DTPA) to the round window niche of 12 guinea pigs using Seprapack ${ }^{\mathrm{TM}}$ (carboxlmethylcellulose-hyaluronic acid) pledgets which stabilized the fluid volume in the round window niche. Gd-DTPA distribution was monitored sequentially with time following application. Distribution in normal, unperforated ears was compared with ears that had undergone a cochleostomy in the basal turn of scala tympani and implantation with a silastic electrode. Results were quantified using image analysis software. In all animals, Gd-DTPA was seen in the lower basal scala tympani (ST), scala vestibuli (SV), and throughout the vestibule and semi-circular canals by $1 \mathrm{~h}$ after application. Although Gd-DTPA levels in ST were higher than those in the vestibule in a few ears, the majority showed higher Gd-DTPA levels in the vestibule than ST at both early and later time points. Quantitative computer simulations of the experiment, taking into account the larger volume of the vestibule compared to scala tympani, suggest most Gd-DTPA (up to 90\%)
\end{abstract}

Correspondence to: S. J. O'Leary · Department of Otolaryngology · University of Melbourne - 32 Gisborne Street, East Melbourne, VIC 3002, Australia. Telephone: +61 39929 8281; email: sjoleary @unimelb.edu.au entered the vestibule directly in the vicinity of the stapes rather than indirectly through the round window membrane and ST. Gd-DTPA levels were minimally affected by the implantation procedure after $1 \mathrm{~h}$. Gd-DTPA levels in the basal turn of scala tympani were lower in implanted animals, but the difference compared to non-implanted ears did not reach statistical significance.

Keywords: perilymph, inner ear, stapes, annular ligament, magnetic resonance imaging, pharmacokinetics

\section{INTRODUCTION}

Preservation of residual inner ear function after cochlear implantation is of increasing importance to facilitate combined electric and acoustic (electroacoustic) stimulation of the ear (Gantz and Turner 2004; Gantz et al. 2005; Gstoettner et al. 2004) and to retain normal vestibular function in the implanted ear. Electroacoustic stimulation has been shown to be associated with improved speech perception, especially in the presence of background noise (Gantz et al. 2005; Turner et al. 2008), and to improve music appreciation for implant recipients (Gantz et al. 2005). The preservation of vestibular function is important for the maintenance of balance, and this is of particular importance for patients with bilateral implants and in the elderly where disequilibrium can lead to falls or a loss of independent living (Enticott et al. 2011).

Preserving inner ear function has been achieved through modification of electrode designs (Gantz and Turner 2004; Lenarz et al. 2006; Gstoettner et al. 
2008; Fraysse et al. 2006), the adoption of new surgical techniques (Lehnhardt 1993; Briggs et al. 2005; Kiefer et al. 2004), and more recently through the use of pharmacological treatments to the inner ear in the peri-operative period. Hearing protection can be achieved in experimental animals undergoing cochlear implantation when glucocorticosteroids are applied to the inner ear (Chang et al. 2009; Eastwood et al. 2010a, b; James et al. 2008; Maini et al. 2009). Recently, the University of Melbourne and Cochlear implant Clinic in Melbourne presented the first clinical evidence in a randomized placebo-controlled trial (RCT) that the incidence of dizziness following cochlear implantation can be reduced by local steroid application prior to implantation (Enticott et al. 2011).

One promising approach for the administration of therapeutic agents to the inner ear is topical application performed immediately before cochlear implant surgery. We have demonstrated that this approach can lead to hearing protection in the guinea pig through the application of a steroid laden polymeric sponge to the round window prior to implantation. The same polymer (carboxylmethylcellulose and hyaluronic acid, Seprapack, Genzyme) was used in the clinical RCT that demonstrated a reduction in post-operative dizziness. While these results are promising, the pharmacokinetics of steroids following their application to the inner ear remain uncertain, so their therapeutic potential may not yet be optimal. In this study, we address two issues of immediate relevance to hearing protection in cochlear implantation that also have wider ramifications for other otologic uses of locally applied drugs, specifically (1) where do intratympanically applied drugs become distributed in the inner ear and (2) what is the influence of cochlear implantation on drug distribution in the inner ear.

One question of major clinical significance is how topically applied drugs affect cochlear function (hearing) compared with vestibular function (balance). It is well established clinically that some aminoglycosides preferentially cause vestibulotoxicity (Nakashima et al. 2000, Wanamaker et al. 1998, Bagger-Sjoback et al. 1990, Aran et al. 1995) with transtympanic gentamicin to treat Meniere's disease reliably achieving a reduction in caloric function while causing a hearing loss in only $2-10 \%$ of cases (Chia et al. 2004). As gentamicin transfer between ST and SV via the helicotrema is an extremely slow process, it was determined that instead, gentamicin distributed to the vestibule by local communication between ST and SV in the basal turn (Salt and Plontke 2005). Analysis of clinical studies showed that only low levels of gentamicin reached cochlear regions responsible for the hearing of speech frequencies (Salt et al. 2008). The slow distribution of substances along the ear has been supported by magnetic resonance imaging (MRI) studies that have demonstrated gadolinium takes hours to diffuse beyond the second cochlear turn (Zou et al. 2005) and by measurements of the spread of chemical markers along ST (Salt and Ma 2001, Salt et al. 2007) which are consistent with distribution being dominated by passive diffusion. The question of the relative distribution of drugs within the cochlea and vestibular system over time has not been assessed quantitatively, which was one of the aims of this study.

The second question addressed here is whether cochlear surgery associated with cochlear implantation alters the intracochlear distribution of drugs that have been applied topically to the inner ear. In the intact cochlea, perilymph flow is almost nonexistent so that diffusion is the main mechanism by which drugs are distributed throughout the ear (Salt and Plontke 2005). Cochlear implantation could potentially alter this situation dramatically. Perforating the bony otic capsule releases perilymph pressure and results in a volume flow between the cochlear aqueduct and the site of the perforation (Salt and Stopp 1979). This displacement of perilymph by cerebrospinal fluid would be expected to cause a "washout" of the drug. Furthermore, electrode insertion mechanically displaces perilymph within scala tympani. Both of these events are likely to influence redistribution of drug that is already present within scala tympani prior to implantation as a result of topical drug therapy.

In the present study, Gd-DTPA has been used as a marker substance, detected by MRI. A number of prior studies have used similar methodology but have only made qualitative assessments of gadolinium distribution in the ear (Zou et al. 2003, 2005, 2010; Counter et al. 2003). In this study, semi-quantitative measurements derived from MR images allowed different regions of the inner ear with different treatments, such as cochlear implantation, to be compared across groups of animals.

\section{MATERIALS AND METHODS}

\section{Animal preparation}

The study was approved by the Royal Victorian Eye and Ear Hospital Animal Ethics Committee (Ethics Approval 09/190AR) and the Howard Florey Institute Animal Ethics Committee (Ethics Approval 09-104). Twelve tri-color adult guinea pigs (Dunkin-Hartley strain) of either sex weighing between 350 and $500 \mathrm{~g}$ were used in the study. The animals were anesthetized with isoflourane mixed with $500 \mathrm{~mL}$ pure air and $500 \mathrm{~mL}$ oxygen during surgery and MRI scanning; $0.05 \mathrm{ml} / \mathrm{kg}$ of Temgesic (Schering-Plough, USA) was administered to inhibit salivation and regurgitation. 
Respiration and temperature were monitored according to HFI SOP-023 during MRI scanning.

\section{Materials}

Ten microliters of paramagnetic gadolinium chelated with diethylenetriamine penta-acetic acid (Gd-DTPA) (Magnevist, concentration $0.5 \mathrm{~mol} / \mathrm{L}$, molecular weight $938 \mathrm{~g} / \mathrm{mol}$, Bayer Schering Pharma AG, Germany) was absorbed into 2-mm disks of the carboxymethylcellulose hyaluronic acid polymer, Seprapack $^{\mathrm{TM}}$ (Genzyme Biosurgery, Framingham) for 1 min prior to round window membrane application.

The cochlear implants used in the study were $100 \%$ biocompatible silastic dummies (sourced from the Department of Otolaryngolgy, University of Melbourne) containing no metallic components. They were cylindrical in shape with diameter $0.4 \mathrm{~mm}$ and length $7.7 \mathrm{~mm}$. The implant was trimmed and inserted $2.25 \mathrm{~mm}$ into the basal turn of scala tympani through a cochleostomy.

\section{Experimental procedure}

Using a dorsolateral posterior-auricular approach, the bulla was opened in each ear using a 1.5-mm diameter drill under a microscope to expose the cochlea. Gdloaded Seprapack pads were placed in the round window niche and remained there for the duration of the experiment. Some ears underwent cochlear implantation after a delay of $30 \mathrm{~min}$ to allow perilymph loading with Gd-DTPA. A basal turn cochleostomy was made approximately $1 \mathrm{~mm}$ from the RWM using a $0.7-\mathrm{mm}$ drill, and the silastic electrode was inserted into ST. After implantation, semi-dried muscle tissue was immediately wrapped around the electrode to reduce perilymph leakage. MRI scans were taken periodically over a 3-h observation period. Both the implants and Seprapack pads remained in place during scanning. Ears were randomly allocated as closed cochlea controls $(n=$ 15 ears) or cochlear implanted ( $n=9$ ears).

\section{Magnetic resonance imaging}

Anesthetized animals were laid supinely on an animal cradle with a pressure sensitive probe positioned under the guinea pig's diaphragm to monitor respiration. Body temperature was monitored with a rectal probe and maintained with an MR compatible electrical heat pad. The cradle was inserted into a BGA12SL gradient set for imaging with a 4.7-T Bruker Biospec 47/30 scanner (Bruker Biospec, Ettlingen, Germany) at the Howard Florey Institute, Australia. A volume coil with uniform sensitivity in the field of view (FOV) was used. The scanning protocol consisted of a three-plan localizer sequence followed by multi-axial, coronal, and sagittal scout images to correctly align a set of six oblique slices through the inner ears that enabled individual turns of the cochlea to be clearly distinguished. Images were then acquired at approximately 12-min intervals with a rapid acquisition, relaxation enhanced (RARE) sequence with the following imaging parameters: recovery time $=$ 2,000 ms; RARE factor $=16$; effective echo time $=$ $38.8 \mathrm{~ms} ; \mathrm{FOV}=3.5 \times 3.5 \mathrm{~cm}^{2}$; reconstructed matrix size $=256 \times 256$; in-plane resolution $=137 \times 137 \mu \mathrm{m}^{2}$; averages $(\mathrm{NEX})=24$; number of slices $=6$; slice thickness $=0.5 \mathrm{~mm}$; and scan time $=9 \min 36 \mathrm{~s}$. This produced predominantly $\mathrm{T} 2$ weighted images, with some T1 shortening, leading to images demonstrating both the labyrinth and also the Gd-DTPA signal. Scanning continued for up to $3 \mathrm{~h}$.

\section{Data analysis}

For low levels of Gd-DTPA, the MRI images show increasing intensity dependent on Gd-DTPA concentration, allowing intensity to be used as a relative measure of Gd-DTPA concentration. For statistical analysis, individual scalae of individual cochlear turns and anterior, oval window and posterior regions in the vestibule were hand selected in the data analysis program on the slice in the image set showing the brightest signal for each region. The intensity of each region was derived from the grayscale image using image analysis software (Image J software, V1.43u, National Institutes of Health, USA). The intensity of each defined region was quantified with a custom written Image J macro that was designed to emphasize the bright, fluid space of the region, while minimizing the influence of the darker, boundary regions. The macro averaged weighted intensity levels of the pixels in a selection. Under this algorithm, the brightest pixel carried a weight of 1 and all other pixels carried a linearly scaled weight according to their intensity, down to pixels at the baseline intensity level which carried a weight of 0 . Thus, a pixel with an intensity that was halfway between baseline and the brightest pixel in the selection carried a weight of 0.5 . This gave an objective intensity measure that was dominated by the brightest pixels in the selection and downweighted the darker, sometimes more numerous, pixels at the periphery of the selection. None of the images analyzed showed saturation of intensity levels.

To account for the variance in MR receiver gain, the images were normalized by dividing the ROI intensities by the average of an area of brain tissue, and subtracting 1 , so that the baseline noise was represented by a value of zero.

Implanted and control ears, diffusion time, and location in the inner ear were compared using paired 
$t$ tests. All calculations were performed using SPSS, Version 17 (IBM software).

Computer simulations of Gd distribution in the inner ear were performed with a new version of the Washington University cochlear fluids simulation program (V3.0). The program has recently been substantially revised and now incorporates all the tissue and fluid spaces of the guinea pig inner ear, including the vestibule and semicircular canals. The new program was made available for download at http://oto.wustl. edu/cochlea/ in August 2010. The program allows the distribution of Gd to be calculated for different entry conditions and locations, taking into account the complex volume and geometry of the inner ear fluid spaces.

\section{RESULTS}

\section{Gd-DTPA distribution}

The scanning plane was orientated to be in alignment with the cochlear modiolus (Fig. 1A). The regions of interest used in the data analysis are shown in Figure. 1B. Application of Gd-DTPA to the round window (RW) niche resulted in an increased intensity in both the vestibule and the cochlea. There was a pronounced decline in intensity towards the apex of the cochlear representing the longitudinal gradient of Gd-DTPA along the cochlear scalae (Fig. 1a).

The raw images, shown as grayscale, and the derived intensity levels for 10 different anatomic regions are shown in Figure 2. Each row of the figure shows serial images from a single non-implanted specimen at $60 \mathrm{~min}$ after Gd-DTPA was applied to the RW niche (Fig. 2A, G, E). Intensity levels derived from the scans of each animal are shown in Figure 2B, $\mathrm{D}$, and $\mathrm{F}$, respectively. In order to identify specific anatomic regions, the anatomic structures in $\mathrm{MR}$ images were compared with a 3D representation of the guinea pig cochlea generated by orthogonal plane fluorescence optical sectioning (shown in Fig. 2H). The 3D cochlea was segmented into slices in a similar orientation to the MR images (shown in Fig. 2G). This allowed the different regions of the cochlear fluid spaces to be identified. At the 1-h measurement point, Gd-DTPA was present in scala tympani, scala vestibuli, the vestibule, and semicircular canals in all ears. The three specimens are shown ranked in terms of relative Gd-DTPA entry into ST. The specimen shown in Figure 2B showed higher intensity levels in the basal part of ST compared to SV, while the specimens in Figure 2D and F showed substantially higher levels in SV compared to the corresponding region of ST. As will be demonstrated below, the results in these latter two animals are not consistent with the sole route of substance entry into the labyrinth being via the RW membrane, with subsequent redistribution from ST to the other perilymphatic regions.

\section{Normal (non-implanted) ears}

The average Gd-DTPA distribution, based on measured intensity levels from 15 control ears, is shown for 1-h post-application (Fig. 3A) and $2.5 \mathrm{~h}$ post-applica-
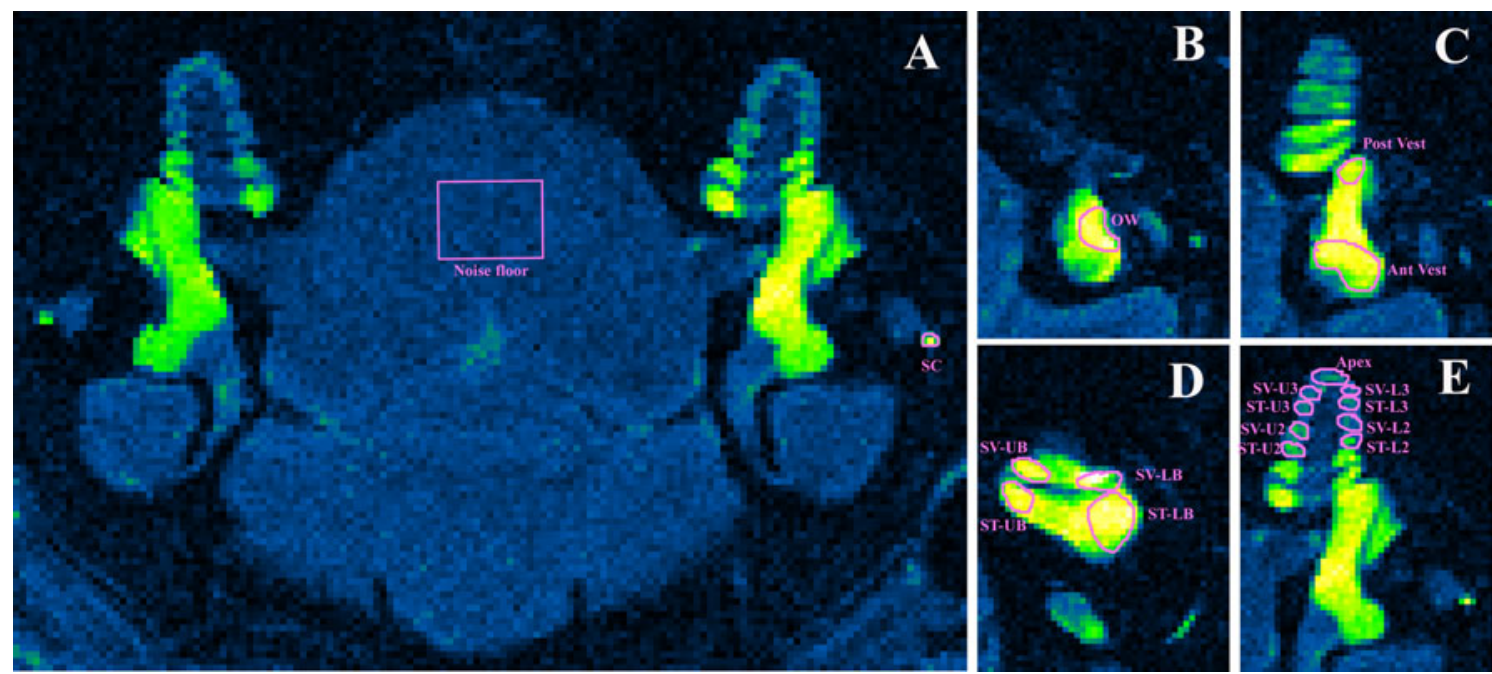

FIG. 1. A Gd-DTPA uptake into the inner ear of the guinea pig. Scan time $32 \mathrm{~min}$; Gd-DTPA application time $3 \mathrm{~h}$ and $7 \mathrm{~min}$; both ears intact, no implantation. B-E The right inner ear with regions of interest (ROI) marked by magenta outlines; OW oval window, Post $V$ posterior vestibule, Ant Vest anterior vestibule, $S V-L B$ scala vestibuli lower basal turn, $S T-L B$ scala tympani lower basal turn, $S V-U B$ scala vestibuli upper basal turn, $S T-U B$ scala tympani upper basal turn, $S V$ - L2 scala vestibuli lower second turn, ST-L2 scala tympani lower second turn, $S V-U 2$ scala vestibuli upper second turn, ST-U2 scala tympani upper second turn, SV-L3 scala vestibuli lower third turn, STL3 scala tympani lower third turn, $S V-U 3$ scala vestibuli upper third turn, $S T-U 3$ scala tympani upper third turn, Apex apical turn. 

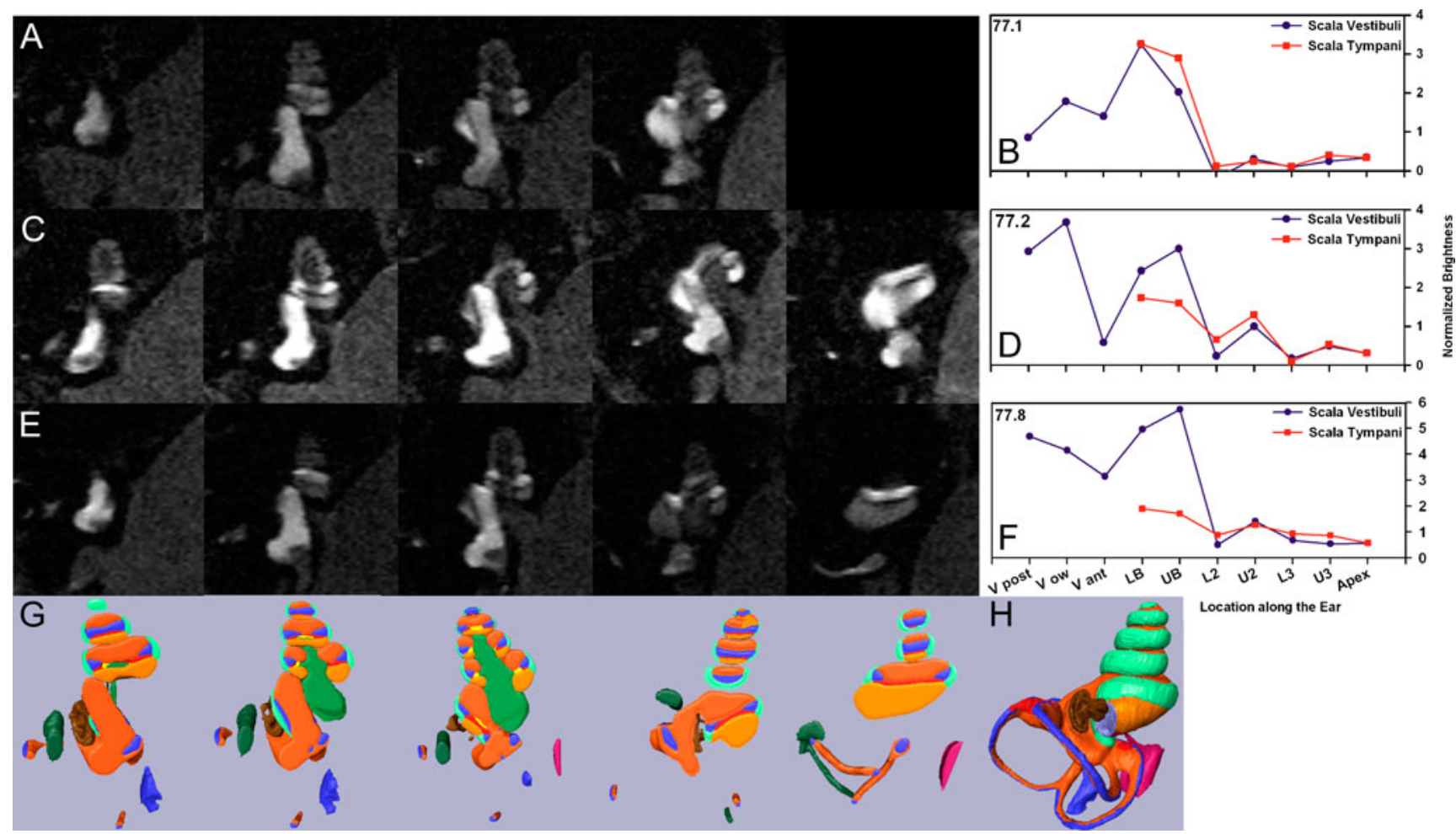

FIG. 2. Measurement and quantification of Gd-DTPA distribution patterns at $60 \mathrm{~min}$ after application to the RW niche. A Serial MR sections of a non-implanted ear in which the brightest region occurs at the basal part of ST. b Quantified brightness levels in ST and SV for the specimen shown in (a). Abscissa represents inner ear location, ordinate represents brightness (see "Materials and methods"). $L B$ lower basal turn; $U B$ upper basal turn; $L 2$ lower second turn; $U 2$ upper second turn; L3 lower third turn; U3 upper third turn; Apex apical turn. C A left, nonimplanted ear showing brightest region in SV. D Brightness levels in ST
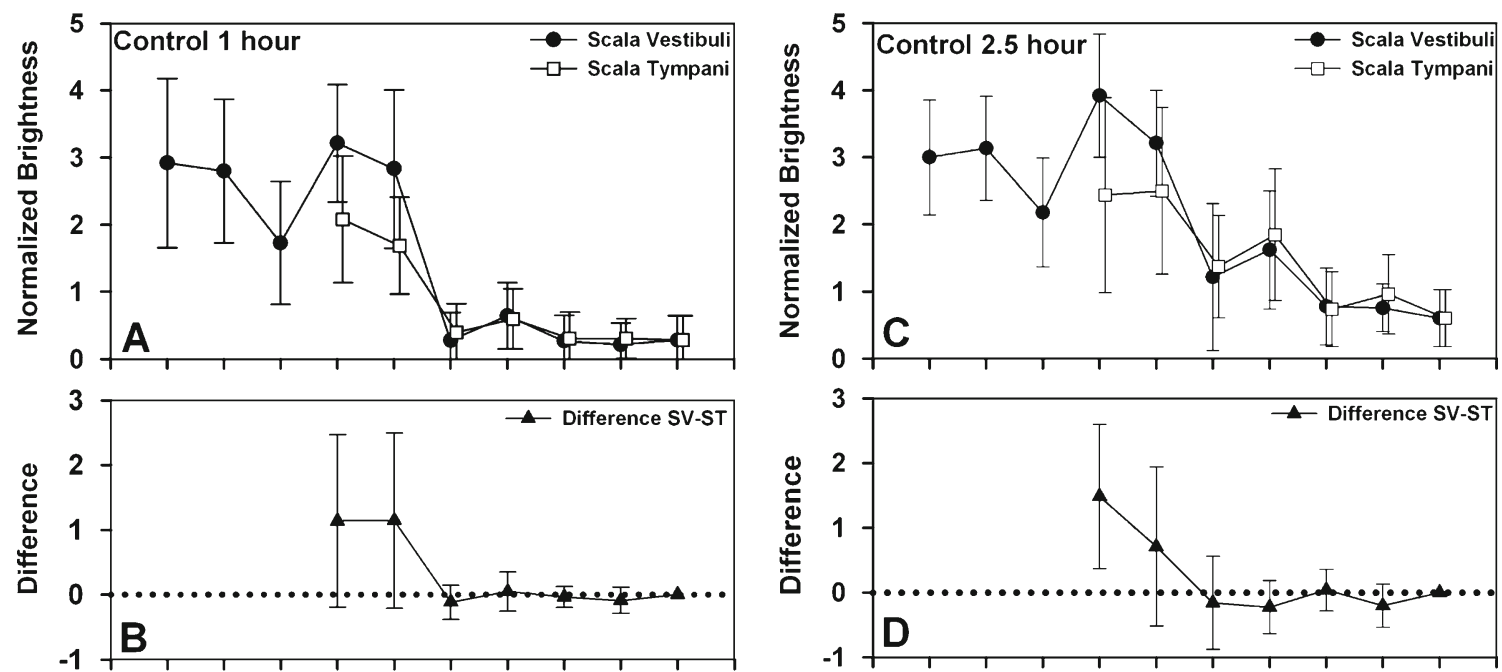

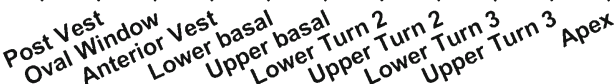

\section{Location along the Ear}

FIG. 3. Average brightness distribution at 10 defined locations in control ears. A $1 \mathrm{~h}$ after Gd-DTPA application to RW niche. Closed dots represent SV, and open squares represent ST. N=15. B Brightness differences between SV and ST after $1 \mathrm{~h}$. In the basal turn, SV is

typically brighter than ST, suggesting higher Gd-DTPA levels there. C $2.5 \mathrm{~h}$ after Gd-DTPA application to RW niche. $N=15$. D Brightness differences between SV and ST after $2.5 \mathrm{~h}$. At this time, SV is significantly brighter than ST in the lower basal turn. 
tion (Fig. 3C). Figure 3B and D shows the paired differences between ST and SV, which were calculated on a per-animal basis and averaged. The signal intensity, averaged across all regions of interest, was higher at $2.5 \mathrm{~h}$ than at $1 \mathrm{~h}$ (mean inner ear 2.81 and 2.22, respectively, $p<0.005$, paired $t$ test).

Within the cochlea, the Gd-DTPA levels, averaged across the upper and lower basal turns, were significantly higher in SV than ST at both $1 \mathrm{~h}$ (means 4.02 and 2.88, respectively, $p<0.005$, paired $t$ test) and $2.5 \mathrm{~h}$ (means 4.56 and 3.46, respectively, $p<0.005$, paired $t$ test), reflecting the fact that SV levels were higher in the majority of ears $(73 \%)$ compared to a minority $(27 \%)$ in which ST levels were higher.

Since the vestibule is in direct continuity with SV, and drug could potentially diffuse freely between these structures, Gd-DTPA levels in the vestibule and lower SV (post-vest, oval window, anterior vest, lower basal, and upper basal) were averaged. These values were significantly higher at 2.5 than at $1 \mathrm{~h}$ (means 4.09 and 3.70, respectively, $p<0.05$, paired $t$ test). Similarly, the levels in the middle cochlea (an average of lower turn 2, upper turn 2, and lower turn 3) were significantly higher at the later time (means 2.17 and 1.45 for 2.5 and $1 \mathrm{~h}$, respectively, $p<0.005$, paired $t$ test), reflecting the redistribution (diffusion) of Gd-DTPA from regions of high concentration to regions of lower concentration. At both 1 and $2.5 \mathrm{~h}$, the anterior vestibule tended to have lower levels than adjacent regions measured. Although this was thought to represent some type of measurement artifact, subsequent calculations with our simulation program also show a similar reduction, as presented and discussed below.
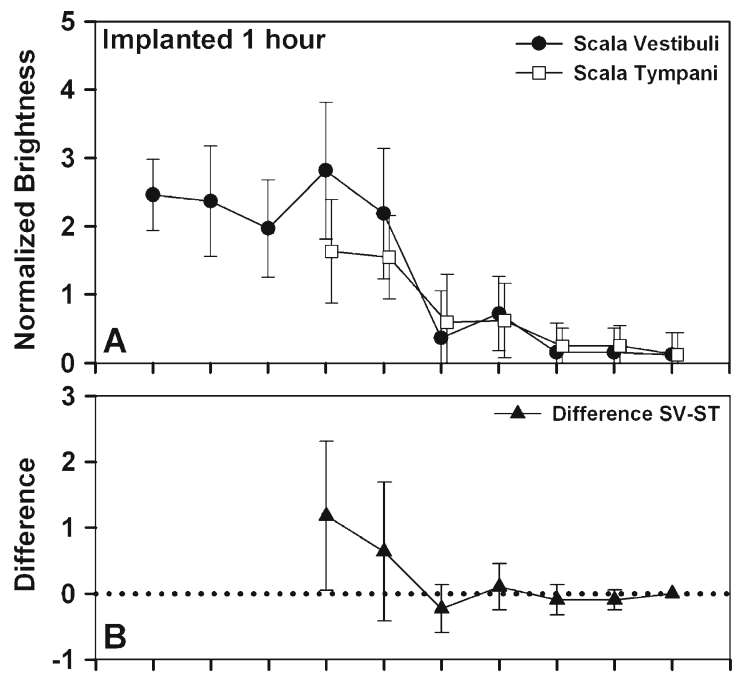

FIG. 4. Average brightness distribution at 10 defined locations in implanted ears. A $1 \mathrm{~h}$ after Gd-DTPA application to RW niche. Closed dots represent SV, and open squares represent ST, N=9. B Brightness differences between SV and ST after $1 \mathrm{~h}$. C $2.5 \mathrm{~h}$ after

\section{The effect of cochlear implantation}

The average Gd-DTPA distribution measured in nine implanted ears is summarized in Figure 4. A surprising feature of these measurements is the observation that the pattern of Gd-DTPA distribution is not influenced markedly by the implantation procedure. Indeed, the differences between control and implanted ears are rather subtle. Levels in the vestibule of implanted ears were generally lower than the controls, and the "dip" in the anterior vestibule was less prominent.

In the majority $(67 \%)$ of implanted labyrinths, Gd-DTPA levels in the basal turn (an average of the upper and lower turns) were again significantly higher in SV than ST at both $1 \mathrm{~h}$ (means 3.50 and 2.59 , respectively, $p<0.005$, paired $t$ test) and at $2.5 \mathrm{~h}$ (means 4.12 and 2.59, respectively, $p<0.005$, paired $t$ test). In the lower second turn, the situation reversed and ST tended to be higher than SV at $2.5 \mathrm{~h}$, which was not apparent in the control.

There was no statistical difference in vestibular levels (average of posterior vestibule, oval window, anterior vestibule) at $1 \mathrm{~h}$ between the implanted and control groups (means 3.27 and 3.48, respectively, $p=0.181, t$ test) or at $2.5 \mathrm{~h}$ (means 3.56 and 3.76 , respectively, $p=0.176, t$ test). Similarly, there was no statistical difference in ST basal turn levels in the cochlea (average of upper and lower basal turn) between implanted and control groups at $1 \mathrm{~h}$ (means 2.59 and 2.96, respectively, $p=0.99, t$ test) but did reach statistical significance at $2.5 \mathrm{~h}$ (means
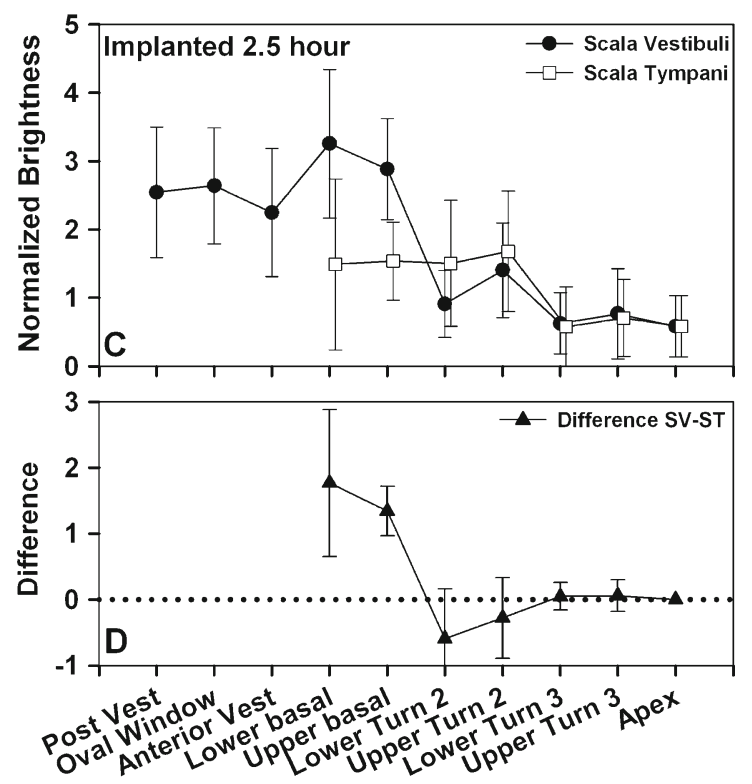

Location along the Ear

Gd-DTPA application to RW niche. Closed dots represent SV, and open squares represent ST. N=9.B Brightness differences between SV and ST after $2.5 \mathrm{~h}$. At this time, both the lower and upper basal turns of $\mathrm{SV}$ are significantly brighter than the adjacent regions of ST. 
2.59 and 3.47, $p<0.05, t$ test), which is consistent with a small loss of Gd-DTPA from the ear at the cochleostomy site.

\section{Computational modeling}

Drug levels in ST and SV were simulated with the recently revised Cochlear Fluids Simulator (V3.0) that incorporates anatomically correct representations of the cochlear fluids spaces, the vestibule, and the semicircular canals. This model included RW entry as an application pathway, but as a result of the data generated here, we also incorporated possible entry of drug into the vestibule at the location of the stapes. Although entry at the stapes was defined by an algorithm similar to that of the RW membrane, based on surface area and permeability values, the area of entry at the stapes is not well defined anatomically. Therefore, the amount of solute entry at the stapes has been presented as a percentage of the combined solute entering through both the RW and stapes routes. Data are shown for zero entry at the stapes (100\% through the RW membrane), $50 \%$ of drug entry at the stapes (50\% through the RW membrane), and $90 \%$ of drug entry at the stapes $(10 \%$ through the RW membrane). The model included communications between ST and the spiral ligament (SL) and between SV and SL, allowing an overall ST-SV communication at a rate comparable to prior marker studies (Salt et al. 1991a, b). While for other drugs, we typically include a rate of elimination to blood; elimination was disabled in the present calculations due to the observed stability of Gd levels indicating a very low rate of elimination. The middle ear concentration used was arbitrary (set to 1,000) and calculated perilymph values are not intended to represent a specific concentration. We chose a middle ear elimination half time of $30 \mathrm{~min}$ based on prior studies (Mikulec et al. 2009) and as this rate reasonably approximated the experimental findings. The calculated distribution of $\mathrm{Gd}$ in the ear when $\mathrm{Gd}$ only enters through the RW membrane is shown in Figure 5A. In this case, the basal turn of ST is initially elevated, followed by a slow decline as the entry rate subsides and drug present becomes distributed both along the cochlea and to SV with time. In this condition, SV concentration levels are always lower than those in ST and tend to be rising with time. The second row of the figure (Fig. 5B) shows the situation when equal amounts of drug enter the RW and in the area of the stapes. Concentration levels in ST again remain substantially higher than those in SV even when equal amounts of drug enter by the two routes. This is primarily because the vestibule, semicircular canals, and SV make up a much larger total volume (approx. $14 \mu \mathrm{L})$ than ST $(6 \mu \mathrm{L})$, so the same amount of solute produces lower concentrations. A larger elevation of SV concentration, comparable to that seen experimentally in control ears, required $90 \%$ of the Gd-DTPA to enter the ear in the stapes area (Fig. 5C). The degree to which drug enters the ear in the stapes area therefore appears to be considerably larger than hitherto appreciated. Also apparent in the calculated profile for SV is a "dip" in the curve at approximately $1.5 \mathrm{~mm}$ from the base (seen as a double-top in the 3D plot Fig. 5C, SV). In the model, this arises from the communication between the vestibule and the middle ear which, when middle ear concentration has fallen below the level in SV, results in an efflux from SV. It is therefore possible that the dip in intensity, observed in the anterior vestibule (Fig. 3A, C), is related to this communication process.

\section{DISCUSSION}

MRI images allow the spatial distribution of Gd-DTPA in inner ear fluids to be quantified. When performed at different times after application, this allows a more complete picture of drug distribution in the ear as a function of distance and time to be established. In previous studies, the technique has generally been used to document the $\mathrm{Gd}$ distribution qualitatively (Zou et al. 2003, 2005, 2010; Counter et al. 2003), but in this study, we have analyzed the data semiquantitatively. The analysis leads us to the novel conclusion that in most ears, the observed distribution of Gd-DTPA cannot be accounted for solely by entry from the RW niche into ST through the RW membrane, with subsequent local redistribution from ST to SV in the basal turn. On the contrary, in most animals, Gd-DTPA levels in the vestibule considerably exceeded those in ST. When the larger volume of the vestibule, SV, and SCC are considered, this can only be accounted for by the conclusion that the majority of Gd-DTPA is entering perilymph into the vestibule directly, possibly via the footplate or annular ligament of the stapes, rather than indirectly via ST.

Establishing the communication routes from the middle ear to the inner ear and between different compartments of the inner ear is complex. Based on the studies with intracochlear injections of markers, it is known that there is local communication between ST and SV (Richardson et al. 2005), in all turns of the cochlea, by solutes passing through the loose cellular structure of the spiral ligament (Salt et al. 1991a, b). It has therefore been assumed that the route of entry of most drugs was through the round window into ST, and subsequent diffusion across the spiral ligament into SV and the vestibule (Salt and Plontke 2005). Based on this explanation, it should not be possible to have a higher drug level in SV relative to ST, except in cases where drug 

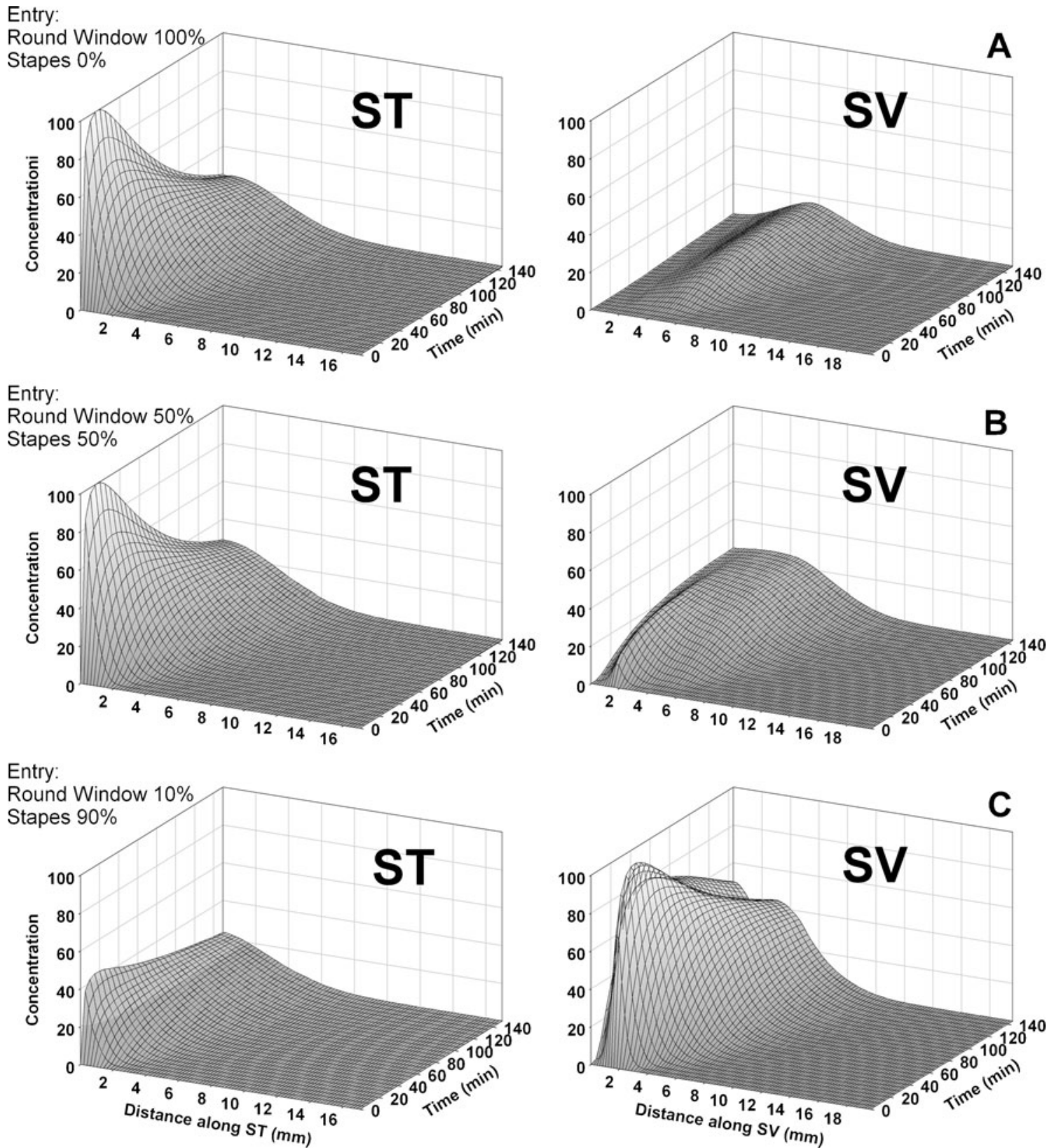

FIG. 5. Calculated Gd concentration profiles as a function of inner ear location and time. Each row (ST and SV, which includes the vestibule) represents a specific condition. A Calculated Gd levels in ST and SV, respectively, when all drug enters through the RW membrane. B Calculated Gd levels in ST and SV, respectively, when $50 \%$ of the drug enters through the RW membrane and $50 \%$ enters

elimination (to blood) occurred at a faster rate from ST than SV. In the case of Gd-DTPA, it is apparent from the similar levels seen at 1 and $2.5 \mathrm{~h}$ post-treatment that the rate of elimination is low, so a rapid elimination of Gd-DTPA from ST cannot account for the present findings. Instead, based on our calculations (summarized in Fig. 5), we can only conclude that Gd-DTPA predominantly enters the inner ear near the stapes, rather than through the RW membrane. The variability in ST and SV levels appears to be dominated by inter animal differences

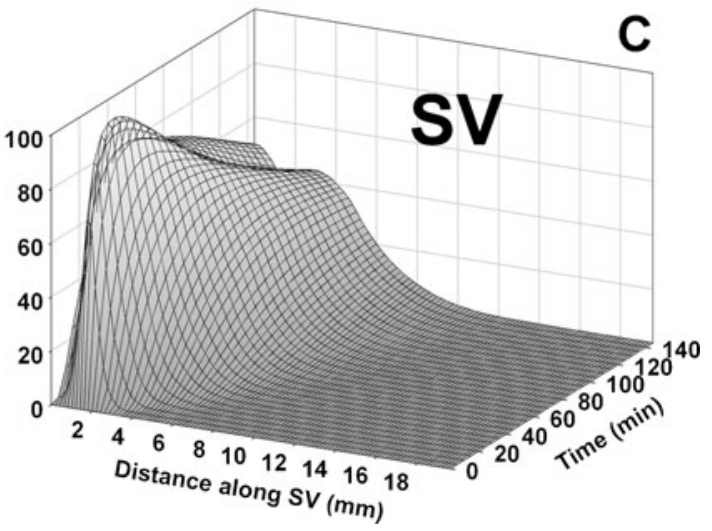

the vestibule in the area of the stapes. SV concentrations remain lower than those in ST because of the larger perilymph volume in the SV compartment relative to ST. C Calculated Gd levels in ST and SV, respectively, when just $10 \%$ of the drug enters through the RWM, and $90 \%$ enters via the stapes. The higher level seen in SV relative to ST is comparable to that found experimentally.

in the amount of Gd-DTPA entering by different routes, rather than by random measurement errors.

Prior studies have reported higher levels in scala vestibuli than scala tympani (Zou et al. 2005, 2010). Several possibilities have been postulated to explain this including a connection to calcium channels (Zou et al. 2005) and the potential additional passage through the annular ligament around the stapes footplate (Zou et al. 2005, 2010); however, no one has yet interpreted their findings quantitatively. 
The conclusion that significant amounts of drug enter the vestibule directly has far-reaching implications for intratympanic applications of drugs in research animals and clinically in humans. It has previously been assumed that intratympanically applied gentamicin suppressed function of otolith organs and SCC with little effect on hearing by spreading locally from ST to the vestibule in the basal turn (Salt and Plontke 2005, Kawai et al. 2010, Naganawa et al. 2008, Nakashima et al. 2000, Watanabe et al. 1971). It was also assumed that to achieve this, the vestibular hair cells were more sensitive to the ototoxic influence of gentamicin than cochlear hair cells (Nakashima et al. 2000, Chia et al. 2004, BaggerSjoback et al. 1990, Wanamaker et al. 1998, Aran et al. 1995). The possibility must now be considered that greater concentrations of gentamicin may be generated in the perilymph of the vestibule and SCC than has previously been appreciated. Similarly, with intratympanic steroids, the distribution and pharmacokinetics are likely to be significantly influenced by the possibility of substantial entry directly into the vestibule.

The independent loading of ST and SV with Gd-DTPA also has significant implications for the quantification of drug dispersion in the cochlea. When drugs only enter through the RW membrane, a significant proportion of the drug in ST diffuses across the spiral ligament into SV, so scala vestibuli represents a substantial "sink" accounting for drug loss from ST. If the drug also enters SV near the stapes, then SV does not represent a sink to the same degree and higher levels of drug will be maintained in ST, allowing drug to spread more quickly along the scala. In practice, prior calculations of drug levels have used RW entry and ST-SV communication rates to match experimentally measured data, so that a reduced loss to SV may involve adjustment of RW entry rates slightly but would not otherwise have a major influence on calculated distribution. So, the direct entry of drug into the vestibule may be an important factor affecting the calculation of drug levels in the vestibule and SV but probably has little influence on drug distribution along ST. This may act to raise the level of therapeutic drugs available for inner ear protection during cochlear implantation.

In the present analysis, in common with most prior MRI studies using Gd as a marker, we have taken intensity readings as an index of Gd-DTPA concentrations in the perilymph. Shahbazi-Gahrouei et al. (2001) showed that in saline samples, brightness followed an almost linear dependence on Gd levels. Although for model systems containing cells (melanoma cells or blood), a more complex relationship existed, it is likely that the perilymphatic fluid spaces of the cochlea most closely approximate a simple saline solution. Therefore, for low concentrations of
Gd, an assumption of linearity may be reasonable, but at high concentrations, it is possible that brightness levels would saturate or even decline. Another difficulty arises in attempting to define an appropriate intensity for perilymph at zero $\mathrm{Gd}$ concentration. The normalization procedure we performed, based on brightness of brain tissue (with no Gd-DTPA present) resulted in low apical perilymph normalized values (less than 0.5) at the 1-h time point when it is unlikely that Gd-DTPA had reached the apex in significant amounts. Thus, the Gd-DTPA concentration cannot be taken to be linearly dependent on the normalized brightness level, and values greater than zero do not necessarily mean that Gd-DTPA was present in appreciable concentration. The measurements presented here should therefore be regarded as only a semiquantitative index of Gd-DTPA concentration. In the future, leaving the contralateral ear untreated with $\mathrm{Gd}$ will permit more rigorous and accurate normalization.

A second important conclusion arising from this study relates to the minor, insignificant changes in Gd-DTPA concentration in cochleae receiving a cochlear prosthesis relative to the non-implanted controls. Prior to this study, it was assumed that if the ear was loaded with Gd primarily through the RW membrane, then a fluid leak affecting the basal turn of ST would dramatically affect perilymph drug levels. The present study shows that this is not the case. If Gd enters the perilymph primarily in the stapes region, loading the vestibule, SV and SCC, drug losses from the basal part of ST play an almost negligible role in the overall drug distribution. Once loaded with Gd, the vestibule can be regarded as a large pool of drug, so that even if drug is lost from the base of ST, it will be replenished by local transfer across the spiral ligament. The smaller volume of the basal region of ST relative to the vestibule, SV and SCC means that drug movements from SV to ST will have little influence on SV concentration. It is noted that the average intensity was lower in the lower and upper basal turn in the implanted ears compared to controls but was higher in lower turn 2 of implanted ears. We interpret this as consistent with a local loss of drug from the basal turn of ST due to CSF leak, but a restriction of the effect of this loss on higher turns due to the implant obstructing ST. Thus, for the second turn, drug diffusing into ST from SV across the spiral ligament is restricted from spreading basally and therefore accumulates apical to the implant. Alternatively, the drug gradient in ST may be altered by fluid movements associated with the actual insertion of the electrode. These findings with Gd-DTPA are consistent with prior observations that we have made concerning the effect of applied $n$-acetyl cysteine (NAC) applied to the round window prior to cochlear implantation (Eastwood et al. 2010a, b). 
In that study, we observed that NAC caused a transient loss of hearing when applied to the round window. The frequency range over which this transient loss was observed was much greater following cochleostomy and/or cochlear implantation, raising the possibility that these surgical interventions may have redistributed the NAC already present within the inner ear towards more apical regions. We conclude that while the implant may cause minor, local disturbances to drug distribution, these are not of great significance relative to the high variability of drug entry characteristics between different animals.

A major issue raised by this study is what anatomic structure(s) control the entry of drug into the vestibule. For the RW membrane, it is generally assumed that the fibrous elements do not impede diffusion and that permeability is limited by the cell membranes and tight junctions of the epithelial cell layer facing the RW niche. In the stapes area, it is possible that the drug enters either through the thin bony regions or through the fibrous annular ligament. However, there is also an epithelial membrane over the middle ear surface and an endostial membrane at the perilymphatic surface. It remains to be determined which of these structures limits permeability, which account for interanimal variations and how sensitive each is to experimental (or surgical) manipulations. The clinical translation of the present finding that Gd-DTPA enters the labyrinth predominantly via the stapes is made more complex by anatomical differences between the stapes in guinea pigs and humans. In humans, the annular ligament bounding the stapes is a continuous, flexible structure, while in guinea pigs, the ligament is comprised of two parts: one bounding the stapes and the other attached to the border of the oval window, with a "slit" between the two, presumably allowing increased stapes mobility (Tanaka and Motomura 1981). In view of this anatomic difference, it could be speculated that Gd entry through the structure could occur more readily in guinea pigs than in humans. However, if Gd-DTPA entry is limited by the membranes bounding the structures, then this anatomical difference may not be of importance. It is apparent that images reported in the study of Zou et al. (2005) show Gd-DTPA levels predominantly in the vestibule and semi-circular canals of humans (Their Figures 5 and 6) within $2 \mathrm{~h}$ after middle ear injection, which suggests that direct entry into the vestibule may also dominate Gd entry in human ears.

The entry of Gd into the ear, while demonstrating fundamental pharmacokinetic processes, cannot be regarded as applicable to all other drugs and substances. Gd does not penetrate membrane-bound structures readily. When applied to perilymph of normal ears, Gd does not enter endolymph (Nakashima et al. 2007; Pyykkö et al. 2010), and when applied to endolymph, it does not enter perilymph (Colletti et al. 2010). Gd remains in the fluid space that it was applied to for $24 \mathrm{~h}$ or longer. In contrast, other drugs such as steroids readily enter endolymph (Parnes et al. 1999) and are cleared rapidly from the cochlear fluids (Bird et al. 2007; Plontke et al. 2008). It remains uncertain whether the predominant entry of Gd in the region of the stapes is a general property of the inner ear, or whether this reflects a characteristic specific to $\mathrm{Gd}$, perhaps related to a very low rate of Gd entry through the RW membrane.

\section{ACKNOWLEDGEMENTS}

This authors wish to thank David Wright, Leigh Johnston and Malcolm Davidson for their technical input; Gordana Kel for her assistance with surgery; Ruth Gill for her assistance with sectioning the $3 \mathrm{D}$ rendered OFPOS image to compare to MRI images, figure preparation, and computational modeling; Mason Betan for writing the image analysis macro for data analysis; Hong Wang and Mary Joy Gleeson from the Howard Florey Institute for their assistance with Magnetic Resonance Imaging; Helen Feng for her assistance with the silastic cochlear implants; Jin $\mathrm{Xu}$ for assistance with animal orientation to view the cochlea; Ian Curthoys for general advice; Elisa Borg and Craig Thomson for providing animal husbandry advice; and Frank Nielson for his assistance making a customized Seprapack cutting tool. This work was supported by two scholarships from the University of Melbourne, research grant from the Royal Victorian Eye \& Ear Hospital, Australia; NHMRC 509206; and NIDCD/NIH DC01368 (AS), and a scholarship from the John \& Betty McCreery trust fund.

\section{REFERENCES}

Aran JM, Chappert C, Dulon D, Erre JP, Aurousseau C (1995) Uptake of amikacin by hair cells of the guinea pig cochlea and vestibule and ototoxicity: Comparison with gentamicin. Hear Res 82:179-183

Bagger-Sjoback D, Bergenius J, Lundberg AM (1990) Inner ear effects of topical gentamicin treatment in patients with Meniere's disease. Am J Otol 11:406-410

Bird PA, Begg EJ, Zhang M, Keast AT, Murray DP, Balkany TJ (2007) Intratympanic versus intravenous delivery of methylprednisolone to cochlear perilymph. Otol Neurotol 28:1124-1130

Briggs RJ, Tykocinski M, Stidham K, Roberson JB (2005) Cochleostomy site: implications for electrode placement and hearing preservation. Acta Otolaryngol 125:870-876

Chang A, Eastwood H, Sly D, James D, Richardson R, O'Leary S (2009) Factors influencing the efficacy of round window dexamethasone protection of residual hearing post-cochlear implant surgery. Hear Res 255:67-72

Chia SH, Gamst AC, Anderson JP, Harris JP (2004) Intratympanic gentamicin therapy for Ménière's disease: A meta-analysis. Otol Neurotol 25:544-552

Colletti V, Mandalì M, Carner M, Barillari M, Cerini R, Pozzi Mucelli R, Colletti L (2010) Evidence of gadolinium distribution from the endolymphatic sac to the endolymphatic compartments of the human inner ear. Audiol Neurotol 15: 353-363 
Counter SA, Zou J, BJelke B, KLason T (2003) 3D MRI of the in vivo vestibulo-cochlea labyrinth during Gd-DTPA-BMA uptake. Auditory and Vestibular Systems 14(13):1707-1712

Eastwood H, Chang A, Kel G, Siy D, Richardson R, O'Leary SJ (2010A) Round window delivery of dexamethasone ameliorates local and remote hearing loss produced by cochlear implantation into the second turn of the guinea pig cochlea. Hear Res 265:25-29

Eastwood H, Pinder D, James D, Chang A, Galloway S, Richardson R, O'Leary S (2010B) Permanent and transient effects of locally delivered n-acetyl cysteine in a guinea pig model of cochlear implantation. Hear Res 259(1-2):24-30

Enticott JC, Eastwood HT, Briggs RJ, Dowell RC, O’Leary SJ (2011) Methylprednisolone applied directly to the round window reduces dizziness after cochlear implantation: A randomized clinical trial. Audiol Neurotol 16:289-303

Fraysse B, Macías ar, Sterkers O, Burdo S, Ramsden R, Deguine O, Klenzner T, Lenarz T, Rodriguez MM, Von Wallenberg E, James C (2006) Residual hearing conservation and electroacoustic stimulation with the nucleus 24 contour advance cochlear implant. Otol Neurotol 27(5):624-633

Gantz BJ, Turner C (2004) Combining acoustic and electrical speech processing: Iowa/nucleus hybrid implant. Acta Otolaryngol 124:344-347

Gantz BJ, Turner C, Gfeller KE, Lowder MW (2005) Preservation of hearing in cochlear implant surgery: Advantages of combined electrical and acoustical speech processing. Laryngoscope 115:796-802

Gstoettner WK, Kiefer J, Baumgartner WD, Pok S, Peters S, Adunka O (2004) Hearing preservation in cochlear implantation for electric acoustic stimulation. Acta Otolaryngol 124:348-352

Gstoettner WK, Van de Heyning P, O'Connor AF, Morera C, Sainz M, Vermeire K, McDonald S, Cavalle L, Helbig S, Valdecasas JG, Anderson I, Adunka OF (2008) Electric acoustic stimulation of the auditory system: Results of a multi-centre investigation. Acta Otolaryngol 128:968-975

James DP, Eastwood H, Richardson RT, O'Leary SJ (2008) Effects of Round Window Dexamethasone on Residual Hearing in a Guinea Pig Model of Cochlear Implantation. Audiol Neurotol 13:86-96

Kawai H, Naganawa S, Ishihara S, Sone M, Nakashima T (2010) MR imaging of the cochlear modiolus after intratympanic administration of Gd-DTPA. Magn Reson Med Sci 9:23-29

Kiefer J, Gstoettner W, Baumgartner W, Pok SM, Tillein J, Ye Q Von ILBERG C (2004) Conservation of low-frequency hearing in cochlear implantation. Acta Otolaryngol 124:272-280

LeHNHARDT E (1993) Intracochlear placement of cochlear implant electrodes in soft surgery technique (article in German). HNO 41:356-359

Lenarz T, Stove T, Buechner A, Paasche G, Briggs R, Risi F, Pesch J, BATTMER RD (2006) Temporal bone results and hearing preservation with a new straight electrode. Audiol Neurotol 11(suppl 1): $34-41$

Maini S, Lisnichuk H, Eastwood H, Pinder D, James D, Richardson RT, Chang A, Connolly T, Sly D, Kel G, O'Leary SJ (2009) Targeted therapy of the Inner Ear. Audiol Neurotol 14:402-410

Mikulec AA, Plontke SK, Hartsock JJ, Salt AN (2009) Entry of substances into perilymph through the bone of the otic capsule following intratympanic applications in guinea pigs: Implications for local drug delivery in humans. Otol Neurotol 30:131-138

Naganawa S, Satake H, Iwano S, Sone M, Nakashima T (2008) Communication between cochlear perilymph and cerebrospinal fluid through the cochlear modiolus visualized after intratympanic administration of Gd-DTPA. Radiat Med 26: 597-602

Nakashima T, Teranishi M, Hibi T, Kobayashi M, Umemura M (2000) Vestibular and Cochlear Toxicity of Aminoglycosides: A review. Acta Otolaryngol 120:904-911
Nakashima T, Naganawa S, Sugiura M, Teranishi M, Sone M, Hayashi H, NaKata S, Katayama N, Ishida IM (2007) Visualization of endolymphatic hydrops in patients with Meniere's disease. Laryngoscope 117:415-420

Parnes LS, Sun AH, Freeman DJ (1999) Corticosteroid pharmacokinetics in the inner ear fluids: an animal study followed by clinical application. Laryngoscope 109:1-17

Plontke SK, Mikulec AA, SAlt AN (2008) Rapid clearance of methylprednisolone after intratympanic application in humans. Otol Neurotol 29:732-733

Pyykkö I, Zou J, Poe D, Nakashima T, Naganawa S (2010) Magnetic resonance imaging of the inner ear in Meniere's disease. Otolaryngol Clin North Am 43:1059-1080

Richardson RT, O'Leary S, Wise A, Hardman J, Clark G (2005) A single dose of neurotrophin-3 to the cochlea surrounds spiral ganglion neurons and provides trophic support. Hear Res 204 $(1-2): 37-47$

SALt AN, Stopp PE (1979) The effect of cerebrospinal fluid pressure on perilymphatic flow in the opened cochlea. Acta Otolaryngol 88:198-202

Salt AN, Ohyama K, Thalmann R (1991A) Radial communication between the perilymphatic scalae of the cochlea. I. Estimation by tracer perfusion. Hear Res 56:29-36

Salt AN, Ohyama K, Thalmann R (1991в) Radial communication between the perilymphatic scalae of the cochlea. II. Estimation by bolus injection of tracer into the sealed cochlea. Hear Res 56:37-43

SALT AN, MA Y (2001) Quantification of solute entry into cochlear perilymph through the round window membrane. Hear Res 154:88-97

SAlt AN, PlontKe SKR (2005) Local Inner ear drug delivery and pharmacokinetics. Hearing Research and Drug Discovery. Drug Discov Today 10:1299-1306

Salt AN, Sirjani DB, Hartsock JJ, Gilll RM, Plontke SK (2007) Marker Retention in the Cochlea Following Injections through the Round Window Membrane. Hear Res 232:78-86

Salt AN, Gill RM, Plontke SK (2008) Dependence of Hearing Changes on the Dose of Intratympanically-Applied Gentamicin: A Metaanalysis Using Mathematical Simulations of Clinical Drug Delivery Protocols. Laryngoscope 118:1793-1800

Shahbazi-Gahrouei D, Williams M, Allen BJ (2001) In vitro study of relationship between signal intensity and gadolinium-DTPA concentration at high magnetic field strength. Australas Radiol 45:298-304

Tanaka K, Mотомura S (1981) Permeability of the labyrinthine windows in guinea pigs. Arch Otorhinolaryngol 233:67-73

Turner CS, Reiss LA, Gantz BJ (2008) Combined acoustic and electric hearing: preserving residual acoustic hearing. Hear Res 242:164-171

Wanamaker HH, Gruenwald L, Damm KJ, Ogata Y, Slepecky N (1998) Dose-related vestibular and cochlear effects of transtympanic gentamicin. Am J Otol 19:170-179

Watanabe Y, Nakajima R, Oda R, Uno M, Naito T (1971) Experimental study on the transfer of kanamycin to the inner ear fluids. Med J Osaka Univ 21:257-263

Zou J, Pyykko I, Bjelke B, Dastidar P, Toppila E (2005) Communication between the Perilymphatic Scalae and Spiral Ligament Visualized by in vivo MRI. Audiol Neurotol 10:145-152

Zou J, Pyykko I, Counter A, Klason T, Bretlau P, Bjelke B (2003) In Vivo Observation of Dynamic Perilymph Formation Using a 4.7 T MRI with Gadolinium as a Tracer. Acta Otolaryngol 123:910-915

Zou J, Ramadan UA, Pуиккö I (2010) Gadolinium uptake in the rat inner ear perilymph evaluated with $4.7 \mathrm{~T}$ MRI: a comparison between transtympanic injection and gelatin sponge-based diffusion through the round window membrane. Otol Neurotol 31:637-641 